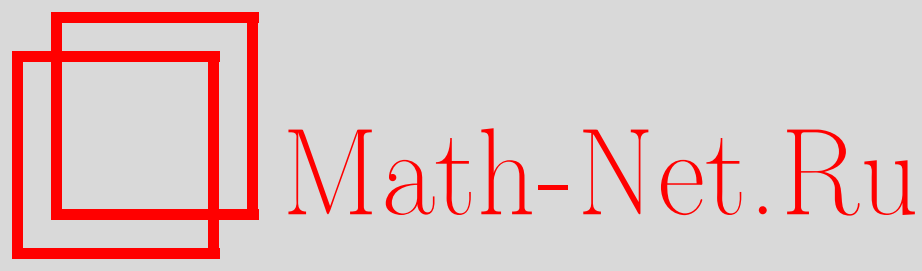

С. С. Герштейн, А. А. Логунов, М. А. Мествиришвили, Невозможность гравитационного коллапса в релятивистской теории гравитации, ТМФ, 2009, том 161, номер 2, 295-304

DOI: https://doi.org/10.4213/tmf6440

Использование Общероссийского математического портала Math-Net.Ru подразумевает, что вы прочитали и согласны с пользовательским соглашением http://www. mathnet.ru/rus/agreement

Параметры загрузки:

IP : 34.229 .45 .116

26 апреля 2023 г., 09:13:32

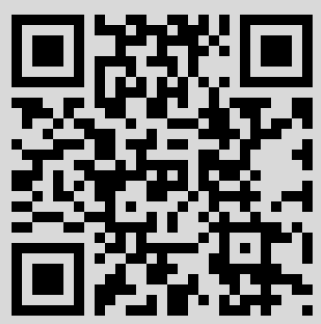




\section{НЕВОЗМОЖНОСТЬ ГРАВИТАЦИОННОГО КОЛЛАПСА В РЕЛЯТИВИСТСКОЙ ТЕОРИИ ГРАВИТАЦИИ}

\footnotetext{
Показано, что согласно релятивистской теории гравитации при отсутствии диссипации пылевой шар будет пульсирующим во времени, а следовательно, коллапс невозможен.
}

Ключевые слова: релятивистский коллапс, черная дыра, общая теория относительности, релятивистская теория гравитации.

Согласно уравнениям общей теории относительности (ОТО) массивная звезда с массой $M>3 M_{\odot}$ после истощения всех термоядерных источников энергии переходит в стадию катастрофического сжатия - наступает коллапс [1]. Этот вывод был сделан авторами работы [1] на основе решений Шварцшильда и Толмена. Именно эта работа послужила позднее основой для вывода о неизбежности гравитационного коллапса и существования "черных дыр" [2].

Однако Эйнштейн тогда же в 1939 г. в статье [3] по сути именно по поводу "черных дыр" (хотя тогда такого названия еще не было) пришел к противоположному физическому выводу: "Основным результатом проведенного исследования является четкое понимание того, что в реальном мире отсутствуют «шварцшильдовские сингулярности»”. И далее: “«Шварцшильдовская сингулярность» отсутствует, так как вещество нельзя концентрировать произвольным образом; в противном случае частицы, образующие скопления, достигнут скорости света". Относительно этого вывода Эйнштейна Прескилл и Торн в 1995 г. писали [4]: "В некотором смысле то, что мы называем теперь черной дырой, уже было известно в 1916 г., когда Карл Шварцшильд нашел свое решение полевого уравнения Эйнштейна. Но в течение десятилетий большая часть физиков упорно сопротивлялась таким «возмутительным» приложениям решения Шварцшильда. (Эта часть физиков включала в себя и самого Эйнштейна, который написал в 1939 г. вызывающую сожаление статью, в которой доказывал, что черные дыры не могут существовать)".

В настоящей работе мы, следуя релятивистской теории гравитации (РТГ), покажем, что пылевой шар будет пульсирующим во времени, и это подтверждает правильность физического вывода Эйнштейна, что в реальном мире отсутствуют

${ }^{*}$ Институт физики высоких энергий, Протвино, Московская обл., Россия. E-mail: Semen.Gershtein@ihep.ru, Anatoly.Logunov@ihep.ru 
«шварцшильдовские сингулярности», а поэтому коллапс невозможен, а следовательно, невозможны и черные дыры.

В РТГ гравитационное поле рассматривается как тензорное физическое поле, развивающееся наряду с другими физическими полями в пространстве Минковского. Гравитационное поле универсально, поскольку его источником является полный сохраняющийся тензор энергии-импульса всех полей материи, включая и гравитационное поле. Свойство универсальности тензорного гравитационного поля приводит к возможности описания явлений в эффективном римановом пространстве. В отличие от ОТО в РТГ риманово пространство не является первичным, оно эффективное и имеет только простую топологию.

Представление гравитационного поля как физического поля в пространстве Минковского приводит к необходимости введения массы покоя гравитона, которая становится элементом теории, связывающим пространство Минковского с эффективным римановым пространством. Именно благодаря этому и возникает связь между степенью замедления времени гравитационным полем и ростом абсолютной величины скалярной кривизны пространства, которая, как и давление, противодействует гравитационному сжатию.

Таким образом, в РТГ гравитационные силы являются не только силами притяжения, но при определенных условиях (в сильных полях) они проявляют себя и как силы отталкивания.

Полную систему уравнений РТГ [5] можно записать в виде уравнений для гравитационного поля

$$
R_{\mu \nu}=\varkappa\left(T_{\mu \nu}-\frac{1}{2} g_{\mu \nu} T\right)+\frac{m^{2}}{2}\left(g_{\mu \nu}-\gamma_{\mu \nu}\right)
$$

и уравнений движения вещества в гравитационном поле

$$
\nabla_{\nu} T^{\nu \mu}=0, \quad T^{\mu \nu}=\left(\rho+\frac{p}{c^{2}}\right) U^{\nu} U^{\mu}-p g^{\mu \nu}, \quad U^{\nu}=\frac{d x^{\nu}}{d s},
$$

где $\varkappa=8 \pi G / c^{2}, G$ - гравитационная постоянная, $m=m_{\mathrm{g}} c / \hbar, m_{\mathrm{g}}$ - масса покоя гравитона, $\nabla_{\nu}$ - ковариантная производная в римановом пространстве с метрикой $g_{\mu \nu}$. В дальнейшем будем полагать $c=1$.

Уравнения (1) и (2) вместе с уравнением состояния вещества образуют полную систему уравнений для определения метрического тензора $g_{\mu \nu}$, плотности вещества $\rho$, давления $p$ и скорости движения $\vec{v}$. Из системы уравнений (1) и (2) следуют уравнения

$$
D_{\nu} \tilde{g}^{\mu \nu}=0,
$$

здесь $D_{\nu}$ - ковариантная производная в пространстве Минковского с метрикой $\gamma_{\mu \nu}$. Эти ковариантные уравнения можно взять вместо уравнений (2). В инерциальной системе в галилеевых координатах уравнения (3) принимают вид гармонических условий $\partial_{\nu} \tilde{g}^{\mu \nu}=0$. Согласно этим условиям гравитон имеет спин 2 и 0.

Уравнения (1) определяют гравитационное поле, создаваемое источником, а уравнения (2) - поведение вещества в этом гравитационном поле. Поскольку все физические поля развиваются в пространстве Минковского, решения уравнений (1) и (2) должны удовлетворять принципу причинности [5], согласно которому все времениподобные векторы в эффективном римановом пространстве при развитии системы в 
соответствии с уравнениями (1), (2) должны оставаться времениподобными и в пространстве Минковского. Уравнения (1) и (2) общековариантны, и в инерциальной системе в галилеевых координатах они форминвариантны относительно преобразований Лоренца.

Метрический тензор $g_{\mu \nu}$ эффективного риманова пространства связан с гравитационным полем $\tilde{\phi}^{\mu \nu}$ соотношением

$$
\tilde{g}^{\mu \nu}(x)=\tilde{\gamma}^{\mu \nu}(x)+\tilde{\phi}^{\mu \nu}(x),
$$

где

$$
\tilde{g}^{\mu \nu}=\sqrt{-g} g^{\mu \nu}, \quad \tilde{\gamma}^{\mu \nu}=\sqrt{-\gamma} \gamma^{\mu \nu}, \quad \tilde{\phi}^{\mu \nu}=\sqrt{-\gamma} \phi^{\mu \nu} .
$$

Рассмотрим интервал для нестатического сферически-симметричного тела в форме

$$
d s^{2}=g_{00} d t^{2}+g_{11} d r^{2}+g_{22} d \theta^{2}+g_{33} d \phi^{2},
$$

где метрические коэффициенты $g_{00}, g_{11}$ и $g_{22}$ являются функциями радиальной переменной $r$ и инерциального времени $t$, а $g_{33}$ зависит также от угла $\theta$. Введем следующие обозначения:

$$
\begin{aligned}
g_{00}(r, t) & =U(r, t), & g_{11}(r, t) & =-V(r, t), \\
g_{22}(r, t) & =-W^{2}(r, t), & g_{33}(r, t, \theta) & =-W^{2}(r, t) \sin ^{2} \theta .
\end{aligned}
$$

Отличные от нуля компоненты тензора $g^{\mu \nu}$ имеют вид

$$
\begin{aligned}
g^{00}(r, t) & =\frac{1}{U}, & g^{11}(r, t) & =-\frac{1}{V}, \\
g^{22}(r, t) & =-\frac{1}{W^{2}}, & g^{33}(r, t, \theta) & =-\frac{1}{W^{2} \sin ^{2} \theta} .
\end{aligned}
$$

Для интервала пространства Минковского

$$
d \sigma^{2}=d t^{2}-d r^{2}-r^{2}\left(d \theta^{2}+\sin ^{2} \theta d \phi^{2}\right),
$$

используя (4), находим инвариант

$$
\gamma_{\mu \nu} g^{\mu \nu}=\frac{1}{U}+\frac{1}{V}+\frac{2 r^{2}}{W^{2}} .
$$

Кроме этого инварианта, существует также инвариант

$$
\gamma^{\mu \nu} g_{\mu \nu}=U+V+\frac{2 W^{2}}{r^{2}}
$$

Эти инварианты не должны быть сингулярными. Отсюда следует, что метрические коэффициенты $U$ и $V$ ограничены и не обращаются в нуль. Метрический коэффициент $U$ определяет степень замедления хода времени в гравитационном поле. При усилении гравитационного поля он уменьшается.

Свертывая уравнение (1) с $g^{\mu \nu}$ и учитывая инвариант, находим общее соотношение

$$
R+8 \pi G T=2 m^{2}-\frac{m^{2}}{2}\left[\frac{1}{U}+\frac{1}{V}+\frac{2 r^{2}}{W^{2}}\right] .
$$


Поскольку обычно рассматриваются уравнения состояния вещества, для которых справедливо неравенство $T \geqslant 0$, на основании (2) имеем $T=\rho-3 p \geqslant 0$. Равенство (5) замечательно тем, что оно устанавливает прямую точную алгебраическую связь скалярной кривизны $R$, плотности $\rho$ и давления $p$ с метрическими коэффициентами $U, V$ и $W$.

Из точного соотношения (5), в частности, следует, что метрический коэффициент $U(r, t)$ вне вещества не может обратиться в нуль, поскольку в противном случае скалярная кривизна $R$ обратилась бы в бесконечность на поверхности радиуса $r$, и было бы невозможно сшить внутреннее решение с решением вне вещества.

При гравитационном сжатии тела величина $U$, определяющая степень замедления времени, уменьшается, и когда правая часть равенства (5) по абсолютной величине становится значительно больше $8 \pi T$, дальнейшее уменьшение $U$ останавливается из-за роста по абсолютной величины скалярной кривизны $R$, которая, как и давление, противодействует сжатию. Совместное действие давления и рост абсолютной величины скалярной кривизны при гравитационном сжатии тела останавливают процесс сжатия. Именно здесь проявляется свойство упругости пространства (или упругости поля).

Внешнее решение нестатического сферически-симметричного тела, описываемого интервалом (4), является статическим [6]. При сжатии тела его материальная поверхность согласно теории относительности не может иметь скорость, равную скорости света, поэтому должна происходить остановка процесса сжатия, т. е. скорость сферической поверхности $\partial W(t, r) / \partial t$ будет равна нулю. Для каждого значения $r$ есть свое время остановки $t(r)$. Как показано в статье [7], остановка материальной поверхности тела произойдет при радиусе шара, превышающем радиус Шварцшильда. Последнее означает, что согласно РТГ нестатическое сберически-симметричное тело, описываемое интервалом (4), под действием силы сжсатия не может уйти под гравитационный радиус Шваришильда. Именно отсюда следует, что гравитационный коллапс невозможен.

Аналогичный вывод согласно теореме Биркгоффа должен иметь место и в ОТО, поскольку скорость поверхности тела, сжимающейся под действием сил притяжения, не может за конечное собственное время достичь скорости света. В РТГ к невозможности коллапса можно прийти, изучая динамику поля внутри тела. Что же происходит с гравитационным полем внутри тела согласно РТГ и ОТО? Ответ на этот вопрос будет дан ниже.

В инерциальной системе в сферических координатах интервал пространства Минковского имеет вид

$$
d \sigma^{2}=d t^{2}-d r^{2}-r^{2}\left(d \theta^{2}+\sin ^{2} \theta d \phi^{2}\right)
$$

Рассмотрим в этой системе координат сферически-симметричный пылевой массивный $\left(M>3 M_{\odot}\right)$ шар, описываемый в эффективном римановом пространстве интервалом

$$
d s^{2}=U(t, r) d t^{2}-V(t, r) d r^{2}-W^{2}(t, r)\left(d \theta^{2}+\sin ^{2} \theta d \phi^{2}\right)
$$


Метрические коэффициенты удовлетворяют общим требованиям Гильберта, которые вытекают из свойств псевдоримановой геометрии и причинности. Для интервала (7) метрические коэффициенты ограничены неравенствами

$$
U(t, r)>0, \quad V(t, r)>0,
$$

а также удовлетворяют неравенствам

$$
U(t, r)<V(t, r), \quad W^{2}(t, r)>r^{2} U(t, r),
$$

которые следуют из принципа причинности РТГ [5]. Следуя монографиям [8], [9], отнесем метрические коэффициенты внутри тела к классу $C^{3}$, т. е. будем считать, что они обладают непрерывными производными первых трех порядков.

Для задачи о массивном пылевом шаре, которую мы намерены рассмотреть, естественно выбрать вместо инерциальной системы синхронную систему координат, которая будет в то же время сопутствующей веществу. Для этой цели совершим преобразования

$$
d t=\dot{t} d \tau+\dot{t} d R, \quad d r=\dot{r} d \tau+\dot{r} d R
$$

Здесь

$$
\dot{r}=\frac{\partial r(\tau, R)}{\partial \tau}, \quad \dot{r}=\frac{\partial r(\tau, R)}{\partial R} \quad \text { и т. д. }
$$

Эти преобразования также не выводят из класса $C^{3}$. Подставляя (10) в интервал $(7)$ и налагая условия

$$
\dot{t}^{2} U-\dot{r}^{2} V=1, \quad U \dot{t} \dot{t}=V \dot{r} \dot{r},
$$

получим интервал $d s^{2}$ в синхронной системе в форме

$$
d s^{2}=d \tau^{2}-A(\tau, R) d R^{2}-W^{2}(\tau, R)\left(d \theta^{2}+\sin ^{2} \theta d \phi^{2}\right),
$$

где

$$
g_{11}=-A=U \hat{t}^{2}-V \dot{r}^{2}, \quad g_{22}=-W^{2}(\tau, R) .
$$

Область изменения переменной $R$ ограничена радиусом тела, область изменения переменной $\tau$ - конечным временем сжатия или временем расширения. Поэтому в замкнутой ограниченной области изменения переменных $\tau$ и $R$ метрические коэффициенты и их производные в силу непрерывности также будут ограничены (теорема Вейерштрасса).

При преобразованиях (10) интервал (6) принимает вид

$$
d \sigma^{2}=\gamma_{00} d \tau^{2}+2 \gamma_{01} d \tau d R+\gamma_{11} d R^{2}-r^{2}\left(d \theta^{2}+\sin ^{2} \theta d \phi^{2}\right),
$$

где

$$
\gamma_{00}=\left(\dot{t}^{2}-\dot{r}^{2}\right), \quad \gamma_{01}=(\dot{t} \dot{t}-\dot{r} \dot{r}), \quad \gamma_{11}=\left(\dot{t}^{2}-\dot{r}^{2}\right), \quad \gamma_{22}=-r^{2} .
$$

С помощью соотношений (11) исключим из метрических коэффициентов (13) и (15) производные $\dot{t}$ и $\dot{t}$. После этих вычислений находим

$$
\begin{gathered}
g_{11}=-A=-\frac{V \dot{r}^{2}}{1+V \dot{r}^{2}}, \quad g_{22}=-W^{2}, \\
\gamma_{00}=\frac{1+V \dot{r}^{2}}{U}-\dot{r}^{2}, \quad \gamma_{11}=\dot{r}^{2}\left[\frac{V^{2} \dot{r}^{2}}{U\left(1+V \dot{r}^{2}\right)}-1\right], \quad \gamma_{22}=-r^{2} .
\end{gathered}
$$


Именно эти выражения нам понадобятся в дальнейшем.

Компоненты тензора вещества в правой части уравнения (1) в синхронной системе координат (12) для пылевого шара $(p=0)$ равны

$$
\begin{aligned}
T_{00}-\frac{1}{2} g_{00} T & =\frac{1}{2} \rho, & T_{11}-\frac{1}{2} g_{11} T & =\frac{1}{2} \rho A, \\
T_{22}-\frac{1}{2} g_{22} T & =\frac{1}{2} W^{2} \rho, & T & =\rho .
\end{aligned}
$$

Обратимся теперь к уравнениям (1) для интервала (12). В этом случае, учитывая (14) и (15), для компонент $\gamma_{00}, \gamma_{11}$ и $\gamma_{22}$ получим следующие уравнения:

$$
\begin{gathered}
-\frac{\ddot{A}}{4 A}+\frac{\dot{A}^{2}}{8 A^{2}}-\frac{\ddot{W}}{W}=\frac{\varkappa \rho}{4}+\frac{m^{2}}{4}\left(1-\gamma_{00}\right), \\
-\frac{\ddot{W}}{W A}+\frac{\dot{A} \dot{W}}{2 W A^{2}}+\frac{\dot{A} \dot{W}}{2 W A}+\frac{\ddot{A}}{4 A}-\frac{\dot{A}^{2}}{8 A^{2}}=\frac{\varkappa \rho}{4}+\frac{m^{2}}{4 A}\left(g_{11}-\gamma_{11}\right), \\
-\frac{W^{\prime \prime}}{W A}+\frac{\dot{A} \dot{W}}{2 W A^{2}}+\frac{\dot{A} \dot{W}}{2 W A}+\frac{1}{W^{2}}-\frac{\dot{W}^{2}}{A W^{2}}+\frac{\dot{W}^{2}}{W^{2}}+\frac{\ddot{W}}{W}=\frac{\varkappa \rho}{2}-\frac{m^{2}}{2}\left(1-\frac{r^{2}}{W^{2}}\right) .
\end{gathered}
$$

Вычитая из уравнения (19) уравнение (20), получим

$$
\begin{aligned}
\frac{\ddot{A}}{4 A}- & \frac{\dot{A}^{2}}{8 A^{2}}-\frac{1}{W^{2}}+\frac{\dot{W}^{2}}{A W^{2}}-\frac{\dot{W}^{2}}{W^{2}}-\frac{\ddot{W}}{W}= \\
& =-\frac{\varkappa \rho}{4}+\frac{m^{2}}{2}\left(1-\frac{r^{2}}{W^{2}}\right)+\frac{m^{2}}{4 A}\left(g_{11}-\gamma_{11}\right) .
\end{aligned}
$$

Складывая получившееся уравнение с уравнением (18) и учитывая (16), находим

$$
\begin{aligned}
-2 W \ddot{W}=( & \left.1-\frac{\dot{W}^{2}}{A}+\dot{W}^{2}\right)+ \\
& +\frac{m^{2} W^{2}}{2}\left(1-\frac{r^{2}}{W^{2}}\right)-\frac{m^{2} W^{2}}{4 U}\left(1-\frac{U}{V}\right)\left(1+2 V \dot{r}^{2}\right) .
\end{aligned}
$$

Здесь $\dot{W}$ - скорость частиц вещества, находящихся на сфере радиуса $R$. В правой части уравнения (22) третий член $\left(m^{2} W^{2} / 2\right)\left(1-r^{2} / W^{2}\right)$ из-за малости $m^{2}$ и ограниченности величин $r$ и $W$ крайне мал и поэтому нами в дальнейшем не будет учитываться.

Так как

$$
d W=\frac{\partial W}{\partial t} d t+\frac{\partial W}{\partial r} d r
$$

то, принимая во внимание (10) и учитывая, что $d W=\dot{W} d \tau+\dot{W} d R$, находим

$$
\dot{W}=\frac{\partial W}{\partial t} \dot{t}+\frac{\partial W}{\partial r} \dot{r}, \quad \dot{W}=\frac{\partial W}{\partial t} \dot{t}+\frac{\partial W}{\partial r} \dot{r} .
$$

Используя эти выражения, а также равенства (11), получим

$$
1-\frac{\dot{W}^{2}}{A}+\dot{W}^{2}=\frac{V-(\partial W / \partial r)^{2}}{V}+\frac{1}{U}\left(\frac{\partial W}{\partial t}\right)^{2}
$$


где $\partial W(t, r) / \partial t$ - скорость частиц вещества, находящихся на сфере радиуса $r$. Учитывая в (22) равенство (24), имеем уравнение

$$
-2 W \ddot{W}=\frac{V-(\partial W / \partial r)^{2}}{V}+\frac{1}{U}\left[\left(\frac{\partial W}{\partial t}\right)^{2}-\frac{m^{2} W^{2}}{4}\left(1-\frac{U}{V}\right)\left(1+2 V \dot{r}^{2}\right)\right] .
$$

Из условия причинности [5] для интервала (7) следуют неравенства (9).

Поскольку в ОТО действует только сила гравитационного притяжения, при массе покоя гравитона, равной нулю, правая часть уравнения (25) положительна (см. далее выражение (33)). Сила отталкивания в уравнении (25) возникает только в РТГ из-за наличия массы покоя гравитона, отличной от нуля. Это уравнение РТГ устанавливает связь между ускорением $\ddot{W}$ для частиц, находящихся на сфере радиуса $R$, и важной физической характеристикой гравитационного поля - функцией $U$, определяющей степень замедления времени гравитационным полем. Уравнение (25) описывает изменение во времени $\tau$ радиуса $W(\tau, R)$ сферической поверхности с фиксированным значением переменной $R>0$. Из правой части уравнения (25) для гравитационного поля следует, что наряду с силой притяжения, которая определяется веществом (плотностью $\rho$ ), благодаря свойству упругости поля присутствует и сила отталкивания, которая связана с функцией $U$, определяющей замедление времени из-за гравитационного поля.

Для анализа характера изменения гравитационного поля тела нам достаточно рассмотреть движение вещества (частиц пыли). Остановимся прежде всего на одном замечании. Согласно [5] уравнение (2) для вещества можно заменить уравнением

$$
D_{\nu} \tilde{g}^{\mu \nu}=\partial_{\nu} \tilde{g}^{\mu \nu}+\gamma_{\alpha \beta}^{\mu} \tilde{g}^{\alpha \beta}=0,
$$

где $\gamma_{\alpha \beta}^{\mu}$ - символы Кристоффеля пространства Минковского с метрикой (6). Поскольку все символы Кристоффеля вида $\gamma_{\alpha \beta}^{0}$ равны нулю, из этого уравнения для метрики (4) можно установить связь между метрическими коэффициентами вида

$$
W^{2}=\sqrt{\frac{U}{V}} q(r)
$$

где $q(r)>0$ при $r>0$. Для отличных от нуля символов Кристоффеля $\gamma_{22}^{1}=-r$, $\gamma_{33}^{1}=-r \sin ^{2} \theta$ имеем уравнение

$$
\frac{\partial}{\partial r}\left(W^{2} \sqrt{\frac{U}{V}}\right)=2 r \sqrt{U V}
$$

Из выражения (26) и уравнения (27) следует, что если функция $W$ не зависит от времени $t$, то функции $U$ и $V$ также не зависят от времени. Это обстоятельство, следующее из условия гармоничности, нами не было замечено при рассмотрении уравнений гравитационного поля в статье [10]. Эффект пульсирующего пылевого шара, как мы увидим ниже, остается, однако это требует рассмотрения интервала вида (4) с тремя метрическими функциями, зависящими от времени. Отсюда следует, что в ОТО при использовании условий гармоничности нельзя описать нестатический сферически-симметричный источник с помощью только двух функций $U(t, W)$, $V(t, W)$. 
Прежде чем приступить к качественному анализу уравнения (25), рассмотрим случай пылевого шара в ОТО, когда в (25) масса покоя гравитона равна нулю. Согласно работе Толмена [11] (см. также книгу [12]) имеем

$$
A=\frac{\dot{W}^{2}}{1+f(R)}, \quad 1+f(R)>0
$$

где $f(R)$ - произвольная функция.

Для описания состояния, когда в начальный момент частицы тела находятся в покое, $\dot{W}(0, R)=0$, необходимо выбрать $f(R)<0$. В соответствии с решением Толмена имеем для квадрата радиальной скорости частицы вещества выражение

$$
\dot{W}^{2}(\tau, R)=f(R)+\frac{F(R)}{W}, \quad 8 \pi G \rho W^{2}=\frac{\dot{F}}{\dot{W}},
$$

где $F(R)$ - произвольная функция, положительная при $R>0$ и равная нулю при $R=0 ; \dot{F}$ и $\dot{W}$ считаются положительными, чтобы исключить возможность пересечения сферических слоев при их радиальном движении [12], а также обеспечить положительность плотности $\rho$.

Для сравнения выражения для $A$ в (16) с решением Толмена (28) надо в (16) заменить $r$ на $W$ и учесть (29). После этого получим

$$
V=\frac{W(\tau, R)}{W-F(R)}
$$

На основании этого соотношения и условий Гильберта (8) очевидно, что в физической области значений $(\tau, R)$ имеет место неравенство

$$
W(\tau, R)>F(R) \quad \text { при } \quad R>0 .
$$

Отсюда следует, что при гравитационном сжатии частицы вещества с определенным значением $R>0$ не достигают центра шара $W=0$, более того, они даже не пересекают сферу с гравитационным радиусом $F(R)$.

Сшивая на поверхности шара $R=R_{0}$ внутреннее решение (30) с внешним решением Шварцшильда $V=W /(W-2 G M)$, находим, что для массы шара имеет место соотношение

$$
2 G M=F\left(R_{0}\right)
$$

Заметим, следуя [12], что подобно тому, как $F\left(R_{0}\right)$ определяет согласно (32) гравитационный радиус шара в целом, так $F(R)$ для любого заданного значения $R$ есть гравитационный радиус части шара, расположенной под сферической поверхностью $R=$ const. Но согласно (31) сферическая поверхность гравитационного радиуса $F(R)$ лежит внутри сферы пылевого шара радиуса $W(\tau, R)$, т. е. $W(\tau, R)>2 G M(R)$. Таким образом никакого коллапса пылевого шара не происходит. 
Учитывая выражение (16) для $A$ и принимая во внимание $(30)$, а также осуществляя замену $r$ на $W$, получим формулу

$$
1-\frac{\dot{W}^{2}}{A}+\dot{W}^{2}=\frac{V-1}{V}=\frac{F}{W} .
$$

Уравнение (25) при $m=0$ с учетом соотношений (24) и (33) принимает вид

$$
-2 W \ddot{W}(\tau, R)=\frac{F(R)}{W},
$$

что соответствует отрицательному ускорению, т. е. действию силы притяжения. Это означает, что из-за сил притяжения сами уравнения Гильберта-Эйнштейна не создают динамических условий, исключающих нефизическую область $W(\tau, R) \leqslant F(R)$. Согласно уравнению гравитационного притяжения (34) происходит коллапс. Таким образом, в ОТО возникает противоречие между условиями (31) и уравнением (34). Причина этого противоречия состоит в том, что согласно ОТО гравитационные силы являются только силами притяжения. Именно благодаря этому и возникает противоречие с теорией относительности.

В РТГ внешнее решение нестатического сферически-симметричного тела, описываемого интервалом (7), является статическим [6].

Так как в статическом поле имеет место равенство $\sqrt{U}=\mathrm{const} \cdot \sqrt{1-v^{2}}$, при гравитационном сжатии тела материальная поверхность тела не может иметь скорость, равную скорости света, поэтому должна происходить остановка процесса сжатия. Эта остановка произойдет при радиусе шара, превышающем радиус Шварцшильда [7]. Поскольку в силу (5) функция $U(t, r)$ не может обратиться в нуль на сфере радиуса $r$ и внутри тела, при гравитационном сжатии согласно условию причинности РТГ (9) частицы вещества, находящиеся на поверхности $W(t, r)$, не могут достичь центра пылевого шара $W=0$.

Во время остановки процесса сжатия скорость материальной поверхности шара равна нулю, $\partial W(t, r) / \partial t=0$, а ускорение $\ddot{W}$ из-за малости величины $U$ в соответствии с уравнением (25) станет положительным, следовательно, согласно РТГ из-за силы гравитационного отталкивания происходит остановка процесса сжатия и наступает процесс обратного движения, т. е. расширение.

Таким образом, при наличии массы покоя гравитона в силу уравнения РТГ (25) мы будем иметь пульсирующий пылевой шар; последнее означает, что коллапс пылевого шара невозможен. Точно такая же физическая ситуация возникает и в том случае, когда давление $p$ отлично от нуля.

Поскольку в наших исходных уравнениях отсутствуют диссипативные члены, нестатический массивный пылевой шар будет пульсирующим во времени. Период пульсации в собственном времени $\tau$ может быть и небольшим. Таким образом, мы видим, что внутри тела собственное гравитационное поле благодаря свойству упругости, определяемой отрицательной скалярной кривизной, само себя ограничивает, что исключает коллапс. Вместо коллапса мы имеем пульсирующую динамическую систему. Отсюда следует, что во Вселенной должны присутствовать холодные объекты больших масс, которые находятся в стадии расширения. 
Все вышеизложенное относилось к сферически-симметричным нестатическим объектам. Именно в этом случае, как показано в работе [6], внешнее гравитационное поле объектов будет статическим, а поэтому возникает ограничение, что радиус тела не может быть меньше радиуса Шварцшильда.

\section{Список литературы}

[1] Дж. Оппенгеймер, Г. Снайдер, “О безграничном гравитационном сжатии”, Алъберт Эйнштейн и теория гравитаиии, Сб. статей, ред. Е. С. Куранский, Мир, М., 1979, 353-361.

[2] Дж. Уилер, Б. Гаррисон, М. Вакано, К. Торн, Теория гравитации и гравитационный коллапс, Мир, М., 1967.

[3] А. Эйнштейн, "О стационарных системах, состоящих из многих гравитирующих частиц и обладающих сферической симметрией”, ст. 119, Собр. научн. трудов, Т. II, Наука, М., 1966, 514-531.

[4] Р. Ф. Фейнман, Ф. Б. Мориниго, У.Г. Вагнер, Фейнмановские лекиии по гравитации, Янус-К, М., 2000.

[5] А.А. Логунов, Релятивистская теория гравитации, Наука, М., 2006.

[6] А. А. Логунов, М. А. Мествиришвили, ЭЧАЯ, 40:1 (2009), 136-143.

[7] А. А. Логунов, М. А. Мествиришвили, ТМФ, 121:1 (1999), 4-24.

[8] В. А. Фок, Теория пространства, времени и тяготения, Наука, М., 1961.

[9] Дж. Л. Синг, Общая теория относительности, ИЛ, М., 1963.

[10] С. С. Герштейн, А. А. Логунов, М. А. Мествиришвили, ТМФ, 155:2 (2008), 244-251.

[11] R. C. Tolman, Proc. Nat. Acad. Sci. USA, 20:3 (1934), 169-176.

[12] Л. Д. Ландау, Е. М. Лифшиц, Теория поля, Физматлит, М., 1988. 